\title{
Spontaneous Bilateral Carotid Artery Dissection Revealing Multifocal Fibromuscular Dysplasia
}

\author{
W. Bnouhanna*, W. Fakir, M. Rahmani, M. Benabdeljlil, S. Aïdi \\ Department of Neurology A and Neuropsychology, Mohamed V University, Hôpital des Spécialités, Ibn Sina University Hospital, \\ Rabat, Morocco \\ Email: *w.bnouhanan@gmail.com
}

How to cite this paper: Bnouhanna, W., Fakir, W., Rahmani, M., Benabdeljlil, M. and Aïdi, S. (2019) Spontaneous Bilateral Carotid Artery Dissection Revealing Multifocal Fibromuscular Dysplasia. Case Reports in Clinical Medicine, 8, 250-257. https://doi.org/10.4236/crcm.2019.89031

Received: August 22, 2019

Accepted: September 17, 2019

Published: September 20, 2019

Copyright (c) 2019 by author(s) and Scientific Research Publishing Inc. This work is licensed under the Creative Commons Attribution International License (CC BY 4.0).

http://creativecommons.org/licenses/by/4.0/

\begin{abstract}
Background: Fibromuscular dysplasia (FMD) is a rare idiopathic segmental non-arteriosclerotic and non-inflammatory arterial disease of small to medium-sized vessels. Although it is considered a benign entity, FMD can lead to potentially severe cerebrovascular complications. We present an atypical clinical case of ischemic stroke (IS) caused by bilateral carotid dissection revealing multifocal FMD. Case Report: A 43-year-old right-handed female with no medical history suddenly developed somnolence with left-sided weakness. Clinical examination revealed left hemiplegia and left central facial paralysis with an NIHSS score: 22. Cerebral tomography (CT) with arterial angiogram revealed abilateralis of the middle cerebral arteries (MCA), a double dissection of the internal carotids (IC), aneurysmal ectasia of the left IC, and aneurysm of the M2 segment of the MCA. Brain magnetic resonance imaging (MRI) with arterial angiography confirmed the bilateral dissection. A cerebral angiography (CA) revealed a radiological pattern of multifocal FMD. The angiography of the aorta and its branches showed adiffuse dysplasia of the renal arteries. The patient was put under antiaggregants. The modified Rankin score (mRS) after three months was three. Conclusion: Cerebrovascular FMD has typical angiographic features, the "string of beads" appearance being the most common. Although the risk of a stroke in patients with cervical FMD is low, it can lead to a severe stroke and the long-term risks are unknown. Patient registries should be encouraged to better understand the mechanisms and evolutionary profile of FMD and alert clinicians to this potentially disabling condition.
\end{abstract}

\section{Keywords}

Fibromuscular Dysplasia, Carotid Disease, Stroke 


\section{Introduction}

Fibromuscular dysplasia (FMD) is a rare idiopathic segmental non-arteriosclerotic and non-inflammatory arterial disease of small to medium-sized vessels. The renal and cervical arteries are the most commonly affected, but all arterial territories can be involved. The cause and pathophysiology of FMD are still unknown. Clinical manifestations of FMD are primarily dependent on the affected vessels. Although FMD is usually considered as a relatively benign disease, cervical FMD can lead to potentially serious cerebrovascular complications. We present an atypical and rare clinical case of ischemic stroke (IS) caused by bilateral carotid dissection revealing multifocal Fibromuscular dysplasia (FMD at internal carotid and renal artery with an intracranial aneurysm).

\section{Case Report}

A 43-year-old right-handed Caucasian female with no medical history and with a low cardiovascular risk presented for three months unusual hemicranias and neck pain with recent aggravation. One week before her hospitalization, she abruptly installed somnolence with weakness of the left side. Neurological examination revealed left hemiplegia, left central facial paralysis, left homonymous hemianopia, and dysarthria with an NIHSS score of 22, while cardiovascular including blood pressure and dermatological examinations were normal.

Cerebral tomography (CT) with arterial angiogram revealed an ischemic stroke (IS) of both the middle cerebral arteries (MCA), a double dissection of the two internal carotids with irregularity of the arterial wall at the level of the middle third of the left internal carotid artery, aneurysmal ectasia of the left internal carotid, and aneurysm less than $10 \mathrm{~mm}$ of the M2 segment of the MCA. Brain magnetic resonance imaging (MRI) with arterial angiography and T1 FAT-SAT cervical axial section confirmed the bilateral dissection of the cervical carotids predominant on the right, associated with bilateral IS in the capsular-lenticular territory and the superficial territory of the right MCA (Figure 1). Neurointerventional radiologist was consulted and acerebral digital subtracted catheter angiography (DSCA) was performed showing radiological patterns of multifocal cervical FMD (string-of-beads aspect) in the left IC with bilateral IC dissection (Figure 2 and Figure 3). CT angiography of the aorta and its branches showed an appearance of diffuse arterial dysplasia of the renal arteries (Figure 4).

The patient was put under antiaggregant therapy. For aneurysm of the M2 segment of the MCA, interventional treatment was not retained for our patient, instead, CT angiography monitoring was proposed. Motor rehabilitation and speech therapy were initiated during her hospitalization and continued after discharge. The follow-up is insured by a cerebrovascular disease specialist. After three months of treatment, the modified Rankin score (mRS) is three.

\section{Discussion}

The prevalence of cervical FMD in the general population is unknown. However, 


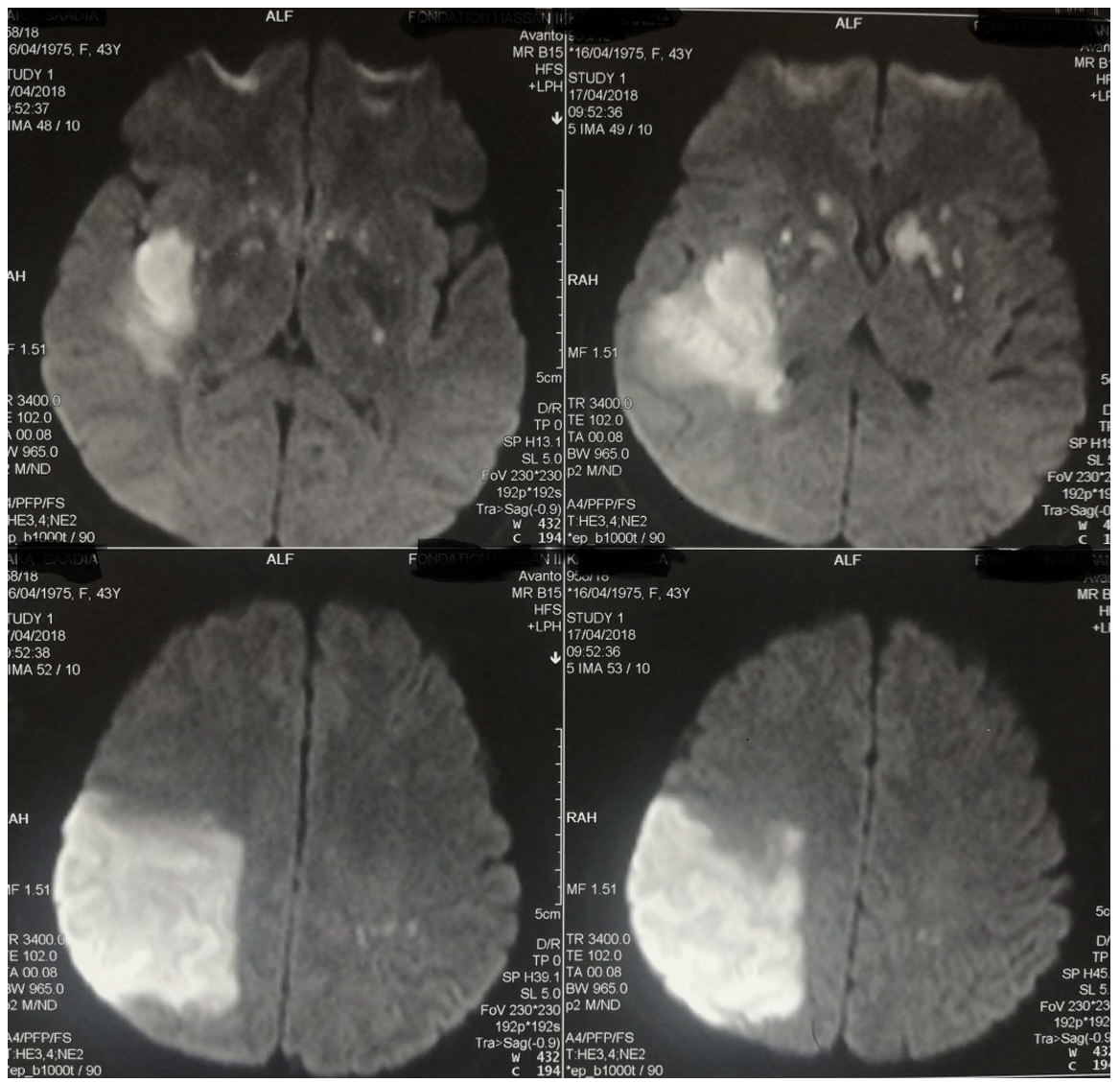

Figure 1. Encephalic MRI, diffusion weighted sequence showing ischemic stroke in the superficial and profound territories of the right ACM MCA and deep territory of the left ACM MCA.

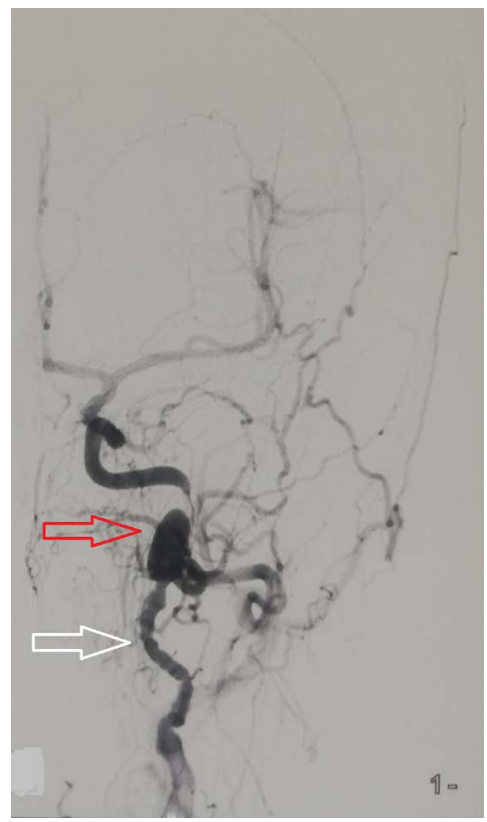

Figure 2. Carotid arteries: digital subtracted catheter angiography. White arrow: Multifocal fibromuscular dysplasia, with typical "string of beads" appearance. Red arrow: Pseudoaneurysm of the petrous segment of the left internal carotid artery. 


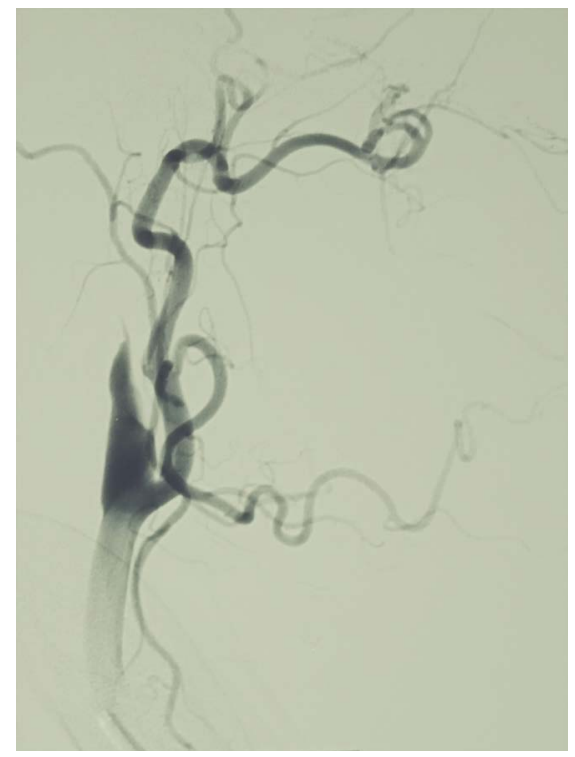

Figure 3. Carotid arteries: digital subtracted catheter angiography, shows a dissection in the right internal carotid artery, with typical "carotid string sign".

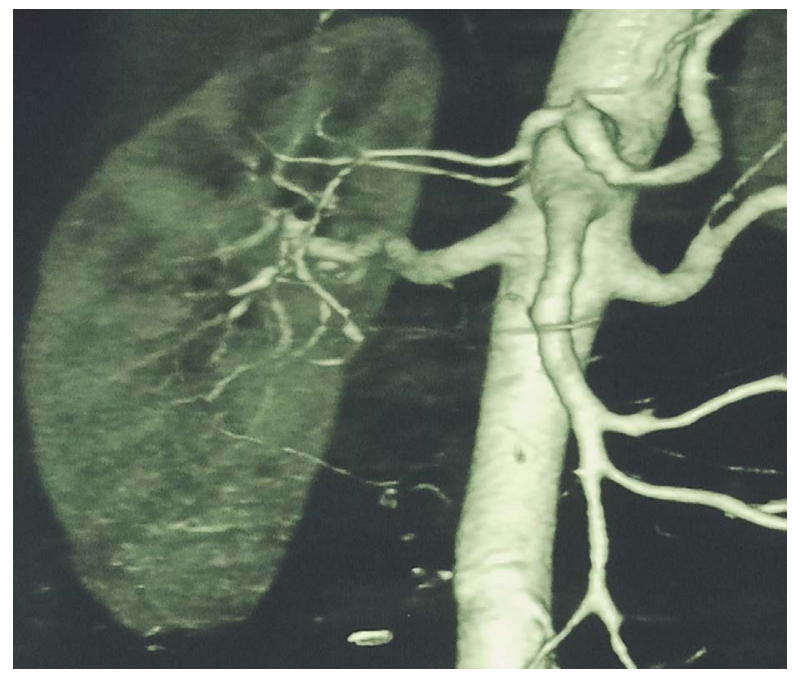

Figure 4. Renal CT angiography; irregular appearance of the distal part of the renal artery arteries.

a study among 20,244 consecutive autopsies performed at the Mayo Clinic over 25 years found only 4 subjects with cervical FMD (0.02\%) [1]. FMD is more common among women while less than $5 \%$ of patients have a family history [2]. The most common mode of revelation is an ischemic or hemorrhagic stroke, TIA, or subarachnoid hemorrhage [3] [4]. The rate of stroke in FMD is 0.75 to $4.9 \%$ [4] [5], which is due to the dissection of the cervical arteries or the rupture of aneurysms [6]. FMD has been identified in approximately $15 \%$ to $20 \%$ of bilateral dissection cases [7]. Mutations in the PHACTR1 (phosphatase and actin regulator 1) gene have recently been associated with cases of spontaneous cervical dissection and cases of DFM [8] [9] [10]. These entities may represent a 
spectrum of the same pathological process.

FMD is often localized in the cervical and renal arteries, hence headaches and pulsatile tinnitus are common when it is manifested by a cervical dissection [11]. In a recent study in the United States, it was shown that one of three patients had FMD in 2 vascular beds [2]. Cerebral arteriography is the gold standard for the diagnosis of FMD. The typical angiographic aspect of a multifocal FMD is the "string of beads" one resulting from the alternation of stenosis with aneurismal dilatation, which is the most common aspect, found in $90 \%$ of cases. This aspect generally affects the medial and distal portions of the internal carotid artery and the V3-V4 segment of the vertebral arteries at the $\mathrm{C} 1$ and $\mathrm{C} 2$ vertebrae, areas usually unaffected by atherosclerosis [12]. In monofocal FMD, unifocal concentric $(<1 \mathrm{~cm}$ in length) or tubular $(\geq 1 \mathrm{~cm})$ smooth narrowing can occur in any portion of the artery [12] [13].

The high prevalence of aneurysms and dissections in other arterial beds (including renal and aortic branch arteries) justify in patients diagnosed with cerebrovascular FMD head-to-pelvic vascular imaging [13].

In the absence of randomized trials, current medical and interventional strategies are based on observational data and expert opinion. The management of ischemic stroke secondary to FMD is similar to that of patients without FMD. In the acute phase, intravenous thrombolysis and/or mechanical thrombectomy are indicated; an antithrombotic agent must be prescribed later [12]. In the case of FMD without stroke complications, including associated asymptomatic cervical artery dissection, daily low-dose aspirin is often indicated to prevent thromboembolic complications. For aneurysm in patients with FMD, similar management as in the general population should be proposed [14]. Securing an unruptured intracranial aneurysm by endovascular coiling or surgical clipping, if embolization is not feasible, must be discussed case by case [5] [12]. Besides, it is recommended to repeat brain magnetic resonance angiography every 5 to 10 years to evaluate the formation of new aneurysms if none have been detected on the baseline imaging (expert opinion).

To our knowledge, there are no prognostic studies in patients with FMD compared to patients with spontaneous cervical dissection. The risk of incident and recurrent stroke in patients with cerebrovascular FMD remains uncertain. Longitudinal studies of patients with cerebrovascular FMD reported a risk of TIA or ischemic stroke during follow-up between $0.6 \%$ and $3.7 \%$ per year and a risk of intracerebral hemorrhage of less than $1 \%$ per year [5]. In the absence of stroke complications, the prognosis of isolated cerebrovascular FMD remains favorable [12]. Our observation is particularly interesting at several levels. First, the case of ischemic stroke due to a bilateral carotid dissection is a scarce situation. Secondly, the carotid dissection reveals a multifocal FMD which is also a rare case. In addition, our observation summarizes the main localizations of the FMD: internal carotid, intracranial aneurysm, and renal artery. Finally, we summarize the latest information about FMD. 


\section{Conclusion}

FMD is a rare vascular disease that affects mainly young women. Renal and cervico-encephalic arteries are the most frequently affected areas. FMD represents a cause of stroke in young people through dissection of the cervical arteries or aneurysm rupture. The string-of-beads aspect is the most common imaging finding of FMD. The management does not differ from that of spontaneous dissection. Patients diagnosed with cerebrovascular FMD should undergo head-to-pelvic vascular imaging. A better knowledge of this disease is critical to shorten the delay to diagnosis and to offer optimal therapeutic management. For that reason, large prospective studies that assess the risk of disease progression, death, and stroke are required to be able to define the best therapeutic strategy.

\section{Conflicts of Interest}

The authors had no financial support when writing this article.

\section{Author's Contributions}

\section{1) Wadi Bnouhanna}

- Conception of the work and Acquisition of data and Analysis of data and Interpretation of data;

- Drafting the work;

- Agree to be accountable for all aspects of the work in ensuring that questions related to the accuracy or integrity of any part of the work are appropriately investigated and resolved.

2) Mounia Rahmani

- Acquisition of data and Analysis of data and Interpretation of data;

- Revising the work critically for important intellectual content;

- Agree to be accountable for all aspects of the work in ensuring that questions related to the accuracy or integrity of any part of the work are appropriately investigated and resolved.

\section{3) Wafa Fakir}

- Acquisition of data.

\section{4) Maria Benabdeljlil}

- Acquisition of data and Analysis of data and Interpretation of data;

- Revising the work critically for important intellectual content;

- Final approval of the version to be published;

- Agree to be accountable for all aspects of the work in ensuring that questions related to the accuracy or integrity of any part of the work are appropriately investigated and resolved.

\section{5) Saadia Aïdi}

- Acquisition of data and/or Analysis of data and/or Interpretation of data;

- Revising the work critically for important intellectual content;

- Agree to be accountable for all aspects of the work in ensuring that questions related to the accuracy or integrity of any part of the work are appropriately 
investigated and resolved.

\section{References}

[1] Schievink, W.I. and Björnsson, J. (1996) Fibromuscular Dysplasia of the Internal Carotid Artery: A Clinicopathological Study. Clinical Neuropathology, 15, 2-6.

[2] Olin, J.W., Froehlich, J., Gu, X., Bacharach, J.M., Eagle, K., Gray, B.H., et al. (2012) The United States Registry for Fibromuscular Dysplasia: Results in the First $447 \mathrm{~Pa}$ tients. Circulation, 125, 3182-3190. https://doi.org/10.1161/CIRCULATIONAHA.112.091223

[3] De Bray, J.M., Marc, G., Pautot, V., Vielle, B., Pasco, A., Lhoste, P. and Dubas, F. (2007) Fibromuscular Dysplasia May Herald Symptomatic Recurrence of Cervical Artery Dissection. Cerebrovascular Diseases, 23, 448-452. https://doi.org/10.1159/000101470

[4] Corrin, L.S., Sandok, B.A. and Houser, O.W. (1981) Cerebral Ischemic Events in Patients with Carotid Artery Fibromuscular Dysplasia. Archives of Neurology, 38, 616-618. https://doi.org/10.1001/archneur.1981.00510100044005

[5] Pasquini, M., Trystram, D., Nokam, G., Gobin-Metteil, M.P., Oppenheim, C. and Touzé, E. (2015) Fibromuscular Dysplasia of Cervicocephalic Arteries: Prevalence of Multisite Involvement and Prognosis. Revue Neurologique, 171, 616-623.

https://doi.org/10.1016/j.neurol.2015.02.011

[6] Kadian-Dodov, D., Gornik, H.L., Gu, X., Froehlich, J., Bacharach, J.M., Chi, Y.W., et al. (2016) Dissection and Aneurysm in Patients with Fibromuscular Dysplasia: Findings from the US Registry for FMD. Journal of the American College of Cardiology, 68, 176-185.

[7] Béjot, Y., Aboa-Eboulé, C., Debette, S., Pezzini, A., Tatlisumak, T., Engelter, S., et al. (2014) Characteristics and Outcomes of Patients with Multiple Cervical Artery Dissection. Stroke, 45, 37-41. https://doi.org/10.1161/STROKEAHA.113.001654

[8] Olin, J.W. (2017) Expanding Clinical Phenotype of Fibromuscular Dysplasia. Hypertension, 70, 488-489. https://doi.org/10.1161/HYPERTENSIONAHA.117.09646

[9] Debette, S., Kamatani, Y., Metso, T.M., Kloss, M., Chauhan, G., Engelter, S.T., et al. (2015) Common Variation in PHACTR1 Is Associated with Susceptibility to Cervical Artery Dissection. Nature Genetics, 47, 78.

[10] Kiando, S.R., Tucker, N.R., Castro-Vega, L.J., Katz, A., D’Escamard, V., Tréard, C., et al. (2016) PHACTR1 Is a Genetic Susceptibility Locus for Fibromuscular Dysplasia Supporting Its Complex Genetic Pattern of Inheritance. PLoS Genetics, 12, e1006367. https://doi.org/10.1371/journal.pgen.1006367

[11] Kadian-Dodov, D., Goldfinger, J.Z., Gustavson, S. and Olin, J.W. (2018) Natural History of Cervical Artery Fibromuscular Dysplasia and Associated Neurovascular Events. Cerebrovascular Diseases, 46, 33-39. https://doi.org/10.1159/000491437

[12] Touzé, E., Southerland, A.M., Boulanger, M., Labeyrie, P.E., Azizi, M., Bouatia-Naji, N., et al. (2019) Fibromuscular Dysplasia and Its Neurologic Manifestations: A Systematic Review. JAMA Neurology, 76, 217-226. https://doi.org/10.1001/jamaneurol.2018.2848

[13] Varennes, L., Tahon, F., Kastler, A., Grand, S., Thony, F., Baguet, J.P., et al. (2015) Fibromuscular Dysplasia: What the Radiologist Should Know: A Pictorial Review. Insights into Imaging, 6, 295-307. https://doi.org/10.1007/s13244-015-0382-4

[14] American College of Cardiology Foundation/American Heart Association Task 
Force (2011) Guideline on the Management of Patients with Extracranial Carotid and Vertebral Artery Disease: Executive Summary. Journal of the American College of Cardiology, 57, 1002-1044. 